\title{
Computer-aided Diagnosis System for the Assessment of Retinal Vascular Changes
}

\author{
Behdad Dashtbozorg ${ }^{1,2}$, Ana Maria Mendonça ${ }^{1,2}$, Susana Penas ${ }^{3,4}$, and Aurélio \\ Campilho ${ }^{2,5}$ \\ 1 INEB - Instituto de Engenharia Biomédica, Porto, Portugal \\ 2 Faculdade de Engenharia, Universidade do Porto, Porto, Portugal \\ 3 FMUP - Faculdade de Medicina Da Universidade do Porto \\ 4 Centro Hospitalar São João, Porto, Portugal \\ ${ }^{5}$ INESC TEC - INESC Science and Technology, Porto, Portugal \\ $\{$ behdad.dashtbozorg, amendon, campilho\}@fe.up.pt
}

\begin{abstract}
This paper presents an automatic application that provides several retinal image analysis functionalities, namely vessel segmentation, vessel width estimation, artery/vein classification and optic disc segmentation. A pipeline of these methods allows the computation of important vessel related indexes, namely the Central Retinal Arteriolar Equivalent (CRAE), Central Retinal Venular Equivalent (CRVE) and Arteriolar-to-Venular Ratio (AVR), as well as various geometrical features associated with vessel bifurcations. The results for AVR estimation were assessed using the images of INSPIRE-AVR dataset; for this dataset, the mean error of the measured AVR values with respect to the reference was identical to the one achieved by a medical expert. The estimation of the CRAE, CRVE and AVR values on 480 images from 120 subjects have shown a significant correlation between right and left eyes and also between images of same eye acquired with different camera fields of view.
\end{abstract}

\section{Introduction}

Among several features associated with vascular changes, the Central Retinal Arteriolar Equivalent (CRAE), Central Retinal Venular Equivalent (CRVE) and Arteriolar-to-Venular Ratio (AVR) have been frequently used as indicators for the early detection, diagnosis, staging and follow-up of some systemic diseases, namely diabetes, hypertension, and vascular disorders, since they can reflect the narrowing or dilation of the retinal blood vessels caused by these diseases $[1$, $2]$. These diseases also change the pattern of vessel branching, thus making the measurement of bifurcation geometrical features a useful tool for the diagnosis or prediction of such pathologic conditions [3].

The main goal of this work was the development of an automatic system for analysing retinal images aiming at the estimation of features useful for the early detection and diagnosis of diabetes, hypertension or cardiovascular pathologies. From the ophthalmologic point of view, this system is also relevant for assessing 


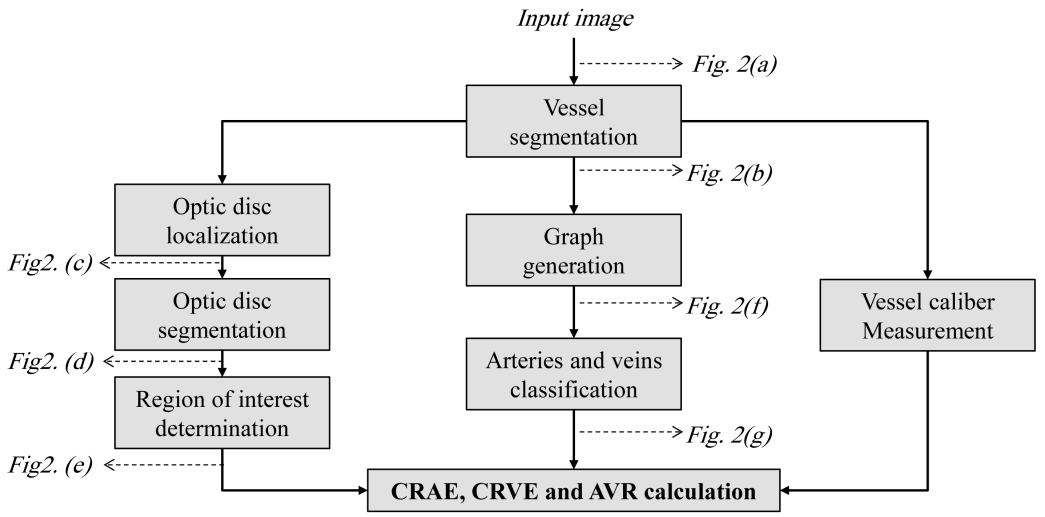

Fig. 1. Block diagram of the methodology for CRAE, CRVE and AVR estimation.

the implications of such diseases in the eye, as well as to evaluate the response of patients to specific therapeutic approaches. Some semi-automatic retinal image analysis systems have been proposed [4], but the development of a fully automatic system for the assessment of vascular changes is still open.

In this paper, we introduce the RetinaCAD System, which is an automated application for a fast and reliable measurement of CRAE, CRVE, AVR values, as well as several geometrical features of retinal vasculature. The evaluation on two distinct image datasets was promising, with results comparable with those achieved by medical experts, showing a low failure rate and a significant correlation between the values calculated for distinct images of both eyes of the same patient.

This paper is organized as follows. Section 2 presents the methods for the segmentation and classification of two main retinal landmarks. The tools available in the framework of the application are described in section 3. Experimental results and conclusions are presented in sections 4 and 5, respectively.

\section{Methodology}

In this section, we briefly describe the methods for the detection and classification of retinal structures which were used for developing the RetinaCAD application. As depicted in Fig. 1, the calculation of CRAE, CRVE and AVR requires vessel segmentation, region of interest determination (ROI), artery/vein (A/V) classification and accurate vessel caliber measurement. Vessel segmentation is used for finding the vessels, and is the primitive stage for vessel caliber estimation and initial optic disc (OD) localization. For segmenting the retinal vessels, the methods previously proposed in [5] and [6] were chosen. Fig. 2(b) illustrates the vascular tree which is obtained from the original image of Fig. 2(a).

The CRAE, CRVE and AVR values are calculated from the calibers of the vessels inside a specific region of interest (ROI), defined as the standard ring area centered on the optic disc center (ODC), within 2 to 3 disc radius from the OD 


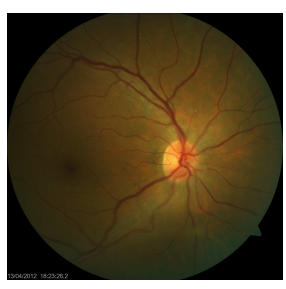

(a)

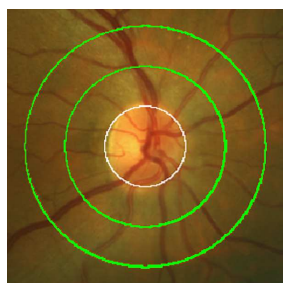

(e)

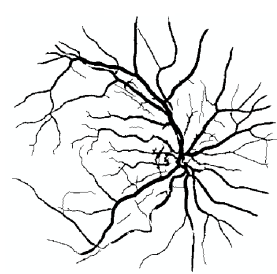

(b)

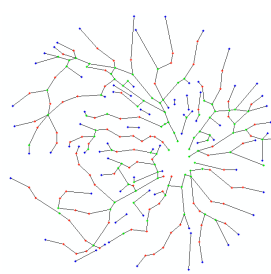

(f)

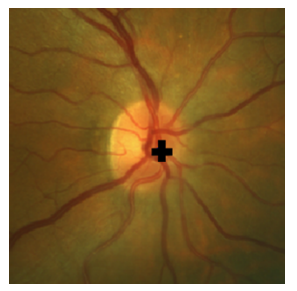

(c)

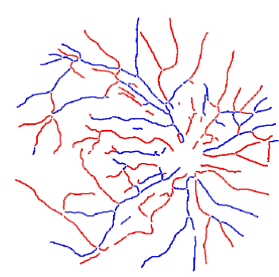

(g)

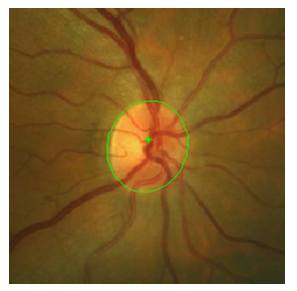

(d)

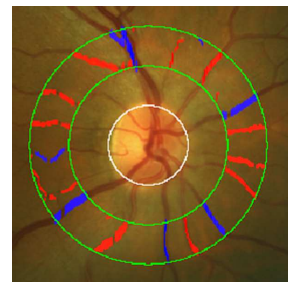

(h)

Fig. 2. (a) Original image; (b) Vessel segmentation result; (c) Initial OD location (black cross); (d) OD segmentation; (e) ROI (delimited by the two green circles) and circular OD margin (white circle); (f) Graph representation of vascular tree; (g) A/V classification result; (h) Arteries and veins inside ROI.

margin [2]. The initial OD location is obtained following the approach described in [7] (Fig. 2(c)), and afterwards used for segmenting the OD boundary based on the application of a multiresolution sliding band filter (SBF) [7]. The final ODC and OD radius are found by fitting a circle to the extracted boundary. Fig. 2(d) and Fig. 2(e) show the final OD boundary and the corresponding ROI for CRAE, CRVE and AVR calculation.

$\mathrm{A} / \mathrm{V}$ classification is based on a graph representation of the retinal vasculature (Fig. 2(f)) as described in [8]. This method classifies the entire vascular tree by deciding on the type of each vessel intersection point (graph nodes) and by assigning one of two labels to each vessel segment (graph links). Final classification of a vessel segment as an artery or a vein is performed through the combination of the graph-based labeling results with a set of intensity features. The result of $\mathrm{A} / \mathrm{V}$ classification for the whole image and inside the ROI are shown in Fig. 2(g) and Fig. 2(h), respectively.

For vessel caliber measurement a distance transform is applied to the segmented vascular tree and the result of this transform in each vessel pixel is its distance to the closest boundary point, $d$. After that, for each vessel centerline pixel, the vessel caliber value, $v c$, is estimated by $v c=2 d-1$.

\subsection{CRAE, CRVE and AVR Computation}

The CRAE and CRVE values are calculated based on Knudtson's revised formula [2]. For computing the CRAE, a set with the six largest arteries inside the 
ROI is first selected. Then, the largest and the smallest vessels in this set are paired up, and the parent trunk width is determined using Knudtson's formula. A new set is formed by substituting the largest and smallest arteries with the parent trunk, and this algorithm is iterated until a single vessel is kept, whose width is the CRAE value. A similar procedure is used for calculating the CRVE. The AVR is defined as the quotient between CRAE and CRVE. For the AVR calculation, the ROI is equidistantly sampled to provide six regions. For each region, the six largest arteries and the six largest veins are identified, and the regional AVR is calculated. The final AVR estimate for the complete image is the average of the six regional values.

\subsection{Computation of the Bifurcation Features}

The branching angles are the basic measurements related to a bifurcation, and these features are obtained from the graph representing the vascular tree, as mentioned before. The branching angles and vessel segment calibers are used to derive features, such as the bifurcation index (quotient between the diameter of the smaller and larger branches), asymmetry ratio (cross-sectional area of the smaller branch divided by that of the larger), diameter ratio (branch diameter divided by trunk diameter), area ratio (sum of cross-sectional areas of two branches divided by that of the trunk) and optimality (deviation of the junction power law value from the optimal value) [3].

\section{RetinaCAD Application}

The RetinaCAD is a fully automatic system for the segmentation and classification of retinal structures and for the measurement of vascular features. This system can analyse OD centered retinal images with variable resolution and camera field of view (FOV). The main retinal image analysis tools provided by this application are: 1) vessel segmentation; 2) vessel centerline extraction; 3) ODC localization; 4) OD segmentation; 5) graph representation of the retinal vascular tree; 6) A/V vessel classification; 7) ROI determination. All tools are fully automatic and have shown a good performance when applied both individually and in combination with each other. The graph representation tool can display local and global features of the links (vessel segments) and nodes (vessel bifurcation/intersection points), such as, caliber and angle between vessel segments and type of intersection. This application automatically measures several global vascular features, namely the CRAE, CRVE, AVR, bifurcation index, asymmetry ratio, diameter ratio, area ratio and optimality.

In order to increase interactivity and usability, we added additional features and tools to this application: 1) option of single click solution for AVR estimation; 2) visualization of intermediate and final results; 3) the ability to process individual task; 4) display of vessel bifurcation/intersection (graph node) features using a mouse click; 5) availability of a follow mouse tool using a second display window for comparison purposes; 6) tools for manual modifications in 


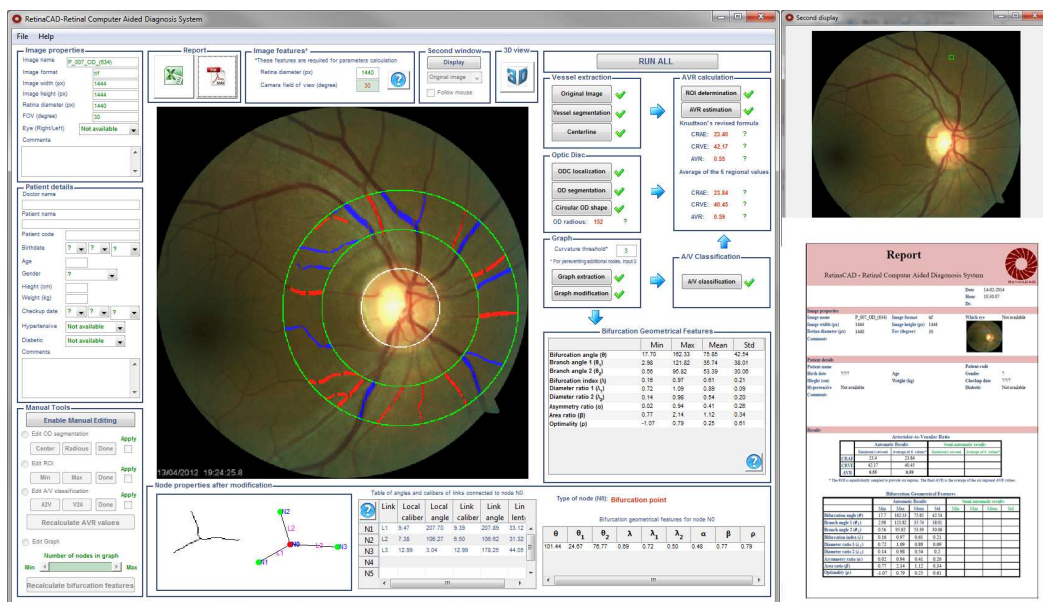

Fig. 3. The interface of the RetinaCAD application and a PDF report example.

ODC location, OD radius value, ROI determination, A/V classification and number of nodes in the graph; 7) generation of reports in PDF or Excel files. Fig. 3 shows the main interface of this application and an example of a PDF report.

\section{Experimental Validation}

This application was evaluated in the images of INSPIRE-AVR dataset [9] and images of a dataset from a local hospital (CHSJ dataset). The forty high resolution images of the INSPIRE-AVR dataset includes two AVR measures that were computed by two ophthalmologists using a semi-automated program, IVAN, developed at the University of Wisconsin [9]. The AVR estimates of Observer 1 are used as reference for calculating the errors for the results of both Observer 2 and our method. The mean error of the measured AVR values with respect to the reference ones was 0.05 which is identical to the one achieved by Observer 2 , thus demonstrating the adequacy of developed application for the automatic calculation of AVR.

The CHSJ dataset contains 528 images from 132 subjects, where for each subject there are four images, two from the right eye and two from the left eye; the two images of each eye were acquired with two different FOV values $\left(45^{\circ}\right.$ and $30^{\circ}$ ). The images of this dataset were used for investigating the robustness of the system to the use of distinct images of the same subject, through the assessment of the correlation between measurements from images of the right and left eyes and from images of same eye with different FOV. For the 528 images that were analysed using the RetinaCAD application, the results for 25 images were not accepted as a consequence of errors in A/V classification or OD segmentation. Although these errors can be solved using the manual modification tool which is included in this application, all the images of the subjects where the automatic procedures have failed (12 subjects) were excluded. In the following, we discuss the results obtained for 480 images from 120 subjects. 
Table 1. Comparison of Mean \pm SD (Min-Max) of measurements for different eyes (right and left) and different FOV $\left(45^{\circ}\right.$ and $\left.30^{\circ}\right)$

\begin{tabular}{|c|c|c|c|c|c|c|c|}
\hline \multicolumn{2}{|c|}{ Measurements } & \multicolumn{4}{|c|}{ RetinaCAD } & \multirow[t]{3}{*}{ Ikram [10] } & \multirow[t]{3}{*}{ Knudtson [2] } \\
\hline & & \multicolumn{2}{|c|}{ Right eye } & \multicolumn{2}{|c|}{ Left eye } & & \\
\hline & & FOV: $45^{\circ}$ & FOV: $30^{\circ}$ & FOV: $45^{\circ}$ & FOV: $30^{\circ}$ & & \\
\hline \multirow{2}{*}{$\begin{array}{l}\text { CRAE } \\
(\mu m)\end{array}$} & & 141.5 & 153 & $146.8 \pm 16.3$ & & & 5.4 \\
\hline & (Min-Max) & $(97.5-172.9)$ & (115.6-177.4) & 184.4) & $(122$ & 35.7) & - \\
\hline \multirow{2}{*}{$\begin{array}{l}\text { CRVE } \\
(\mu m)\end{array}$} & Mear & $237.9 \pm 21.6$ & $255.5 \pm 21.7$ & $239.3 \pm 22.6$ & $259.3 \pm 21.4$ & $222.0 \pm 20.9$ & $242.1 \pm 22.8$ \\
\hline & (Min & (173.9-287.0) & (192.5-303.7) & (168.5-304.2) & $(206.8-314.4)$ & $(135.1-313.6)$ & - \\
\hline \multirow{2}{*}{ AVR } & Mean \pm SD & $0.60 \pm 0.07$ & $0.61 \pm 0.07$ & $0.62 \pm 0.07$ & $0.62 \pm 0.06$ & $0.66 \pm 0.06$ & $0.69 \pm 0.06$ \\
\hline & (Min-Max) & $(0.42-0.82)$ & $(0.41-0.80)$ & $(0.44-0.81)$ & $(0.47-0.79)$ & $(0.48-1.02)$ & - \\
\hline
\end{tabular}

Table 2. Comparison of Mean \pm SD and Pearson correlation between images of the right and left eyes

\begin{tabular}{ccccccc}
\hline \multirow{2}{*}{ Measurements } & \multicolumn{2}{c}{ Mean \pm SD } & \multicolumn{2}{c}{ RetinaCAD } & Le [11] & Leung [12] \\
& Right eye & Left eye & Mean error \pm SD & Corr. & Corr. & Corr. \\
\hline \hline CRAE $(\mu \mathrm{m})$ & $148.0 \pm 14.4$ & $156.1 \pm 12.9$ & $10.0 \pm 7.5$ & 0.71 & 0.71 & 0.7 \\
CRVE $(\mu \mathrm{m})$ & $246.7 \pm 23.4$ & $248.8 \pm 24.0$ & $13.8 \pm 11.4$ & 0.72 & 0.74 & 0.77 \\
AVR & $0.61 \pm 0.07$ & $0.62 \pm 0.07$ & $0.06 \pm 0.04$ & 0.51 & 0.49 & 0.54 \\
\hline Corr: Pean
\end{tabular}

Corr.: Pearson's correlation coefficient.

Table 1 shows the mean and the standard deviation (SD) of the CRAE, CRVE and AVR values for the right and left eyes with different FOV. The values presented by Ikram et al. [10] for a set of 5674 subjects and the ones obtained by Knudtson et al. [2] for a set of 4226 subjects are also included in the two last columns of this table.

The mean error and the Pearson correlation coefficient between measurements from the right and left eyes in the CHSJ dataset are shown in Table 2, where the correlation coefficients reported by the Beaver Dam Eye Study (BDES) [11] and Leung et al. [12] are also included. These results show a good correlation between right and left eyes for CRAE and CRVE and a moderate correlation for AVR, being similar to those reported in [11] and [12]. The agreement between measurements from the right and left eyes can be observed in the Bland-Altman plots depicted in Fig. 4. Each plot shows the mean of two values against the difference between them and permits a visual assessment of the distribution of errors. From the observation of these plots, it is worth mentioning that the results do not show a substantial bias as the mean of differences between the values is small, which demonstrate a good agreement between the results for the two eyes.

In order to evaluate the robustness of the methods in RetinaCAD, we have compared the results of the CRAE, CRVE and AVR values in images of the same eye that were acquired with a different FOV $\left(45^{\circ}\right.$ and $\left.30^{\circ}\right)$. A mean error of 5.9 pixels $(37.6 \mu \mathrm{m})$ and a relative error of $4 \%$ was achieved for the estimated OD radius when the $45^{\circ} \mathrm{FOV}$ and $30^{\circ} \mathrm{FOV}$ images were compared. For the same set of images, the Pearson correlation coefficient and mean error for the CRAE, CRVE and AVR values are shown in Table 3. As can be observed, all indicators show a small mean error and there is a significant correlation between images of the same eye that were acquired in distinct conditions, thus indicating the good performance and consistency of RetinaCAD. 


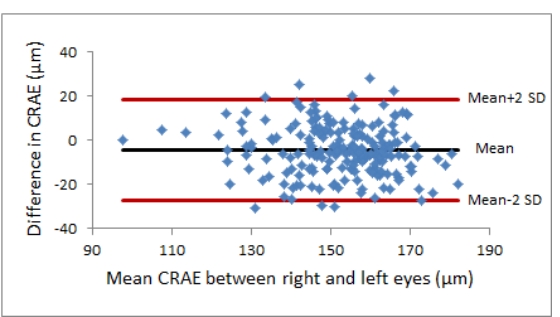

(a)

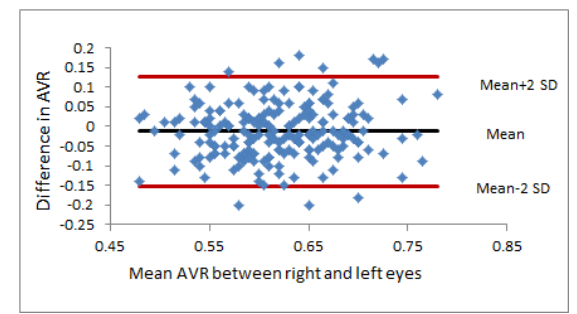

(b)

Fig. 4. Bland-Altman plots of agreement between measurements from the right and left eyes; (a) CRAE; (b) AVR.

Table 3. Comparison of results between images of the same eye with $45^{\circ}$ and $30^{\circ} \mathrm{FOV}$

\begin{tabular}{ccccc}
\hline \multirow{2}{*}{ Measurements } & \multicolumn{2}{c}{ Mean \pm SD } & Mean error \pm SD & \multirow{2}{*}{ Correlation coefficient } \\
& FOV: $45^{\circ}$ & FOV: $30^{\circ}$ & & 0.79 \\
\hline \hline CRAE $(\mu m)$ & $144.3 \pm 15.5$ & $156.1 \pm 12.9$ & $12.7 \pm 8.7$ & 0.79 \\
CRVE $(\mu m)$ & $238.9 \pm 22.3$ & $257.3 \pm 21.9$ & $19.8 \pm 12.1$ & 0.67 \\
AVR & $0.61 \pm 0.07$ & $0.61 \pm 0.07$ & $0.04 \pm 0.04$ & \\
\hline
\end{tabular}

Table 4. The average running time of each individual process in RetinaCAD

\begin{tabular}{|c|c|c|c|c|c|c|c|c|c|c|}
\hline \multirow[t]{2}{*}{ Dataset } & \multirow[t]{2}{*}{ Image size } & \multirow[t]{2}{*}{ FOV } & \multicolumn{8}{|c|}{ Process time (second) } \\
\hline & & & $\begin{array}{l}\text { Vessel } \\
\text { segm. }\end{array}$ & $\begin{array}{c}\text { OD } \\
\text { local. }\end{array}$ & $\begin{array}{l}\text { OD } \\
\text { seg. }\end{array}$ & $\begin{array}{l}\text { ROI } \\
\text { deter. }\end{array}$ & $\begin{array}{c}\text { Graph } \\
\text { gen. }\end{array}$ & $\begin{array}{l}\text { A/V } \\
\text { class. }\end{array}$ & $\begin{array}{l}\text { Features } \\
\text { comput. }\end{array}$ & Total \\
\hline$\overline{I N S P I R E-A V R}$ & $2392 \times 2048$ & $30^{\circ}$ & $\overline{6.38}$ & $\overline{5.37}$ & 3.74 & $\overline{0.49}$ & 1.50 & $\overline{4.91}$ & $\overline{3.76}$ & 26.15 \\
\hline \multirow{2}{*}{ CHSJ } & $1620 \times 1444$ & $30^{\circ}$ & 3.47 & 4.30 & 3.34 & 0.44 & 1.44 & 3.28 & 3.22 & 19.49 \\
\hline & $2196 \times 1958$ & $45^{\circ}$ & 8.86 & 12.54 & 3.89 & 0.79 & 4.51 & 11.72 & 6.95 & 49.26 \\
\hline
\end{tabular}

The RetinaCAD was evaluated on an Intel CPU i7-2600k, $3.40 \mathrm{GHz}, 8 \mathrm{~GB}$ RAM computer. The average running time of each individual process for the images of INSPIRE-AVR and CHSJ datasets are shown in Table 4.

\section{Conclusions}

We have developed a user-friendly system that is able to automatically detect, measure and classify two main retinal landmarks, the optic disc and the vessels. This application can measure several vascular features recognized as indicators for some prevalent systemic diseases. The developed application was assessed in the images of the INSPIRE-AVR dataset where it has achieved a mean error for AVR values identical to the one obtained by a human expert. The low error is promising and demonstrates the reliability of system for AVR calculation.

We have also demonstrated that the correlation between right and left eyes was good for the CRAE and CRVE values, which suggests that the measurements from one eye can provide adequate information about the changes in vessel calibers. After comparing the measured values for images of the same eye but with different FOV, a significant correlation and a low mean error were achieved, thus allowing the conclusion that the RetinaCAD system is adequate both for research and for general clinical use. 
Acknowledgments. This work was supported by FEDER funds through the Programa Operacional Factores de Competitividade-COMPETE and by Portuguese funds through FCT-Fundação para a Ciência e a Tecnologia in the framework of the project PEst-C/SAU/LA0002/2013 and the research grant SFRH /BD/73376/2010.

\section{References}

1. Patton, N., Aslam, T., MacGillivray, T., Pattie, A., Deary, I.J., Dhillon, B.: Retinal vascular image analysis as a potential screening tool for cerebrovascular disease: a rationale based on homology between cerebral and retinal microvasculatures. Journal of anatomy 206(4) (2005) 319-348

2. Knudtson, M.D., Lee, K.E., Hubbard, L.D., Wong, T.Y., Klein, R., Klein, B.E.K.: Revised formulas for summarizing retinal vessel diameters. Current eye research 27 (2003) 143-149

3. Al-Diri, B., Hunter, A., Steel, D., Habib, M.: Manual measurement of retinal bifurcation features. In: in Proc. Engineering in Medicine and Biology Society (EMBC). (January 2010) 4760-4764

4. Lau, Q.P., Lee, M.L., Hsu, W., Wong, T.Y.: The singapore eye vessel assessment system. In: Image Analysis and Modeling in Ophthalmology. CRC Press (2014) $143-160$

5. Mendonça, A., Campilho, A.: Segmentation of retinal blood vessels by combining the detection of centerlines and morphological reconstruction. IEEE Trans. Med. Imag. 25 (September 2006) 1200-1213

6. Mendonça, A., Dashtbozorg, B., Campilho, A.: Segmentation of the vascular network of the retina. In Ng, E.Y.K., Acharya, U.R., Suri, J.S., Campilho, A., eds.: Image Analysis and Modeling in Opthalmology. CRC Press (2014) 85-109

7. Mendonça, A., et al.: Automatic localization of the optic disc in retinal images based on the entropy of vascular directions. In: Image Analysis and Recognition. Volume 7325 of Lecture Notes Comput. Sci. (2012) 424-431

8. Dashtbozorg, B., Mendonça, A., Campilho, A.: An automatic graph-based approach for artery/vein classification in retinal images. IEEE Trans. on Imag. Process. 23(3) (March 2014) 1073-1083

9. Niemeijer, M., Xu, X., Dumitrescu, A., Gupta, P., van Ginneken, B., Folk, J., Abramoff, M.: INSPIRE-AVR: Iowa Normative Set for Processing Images of the Retina-Artery Vein Ratio, http://webeye.ophth. uiowa.edu/component/k2/item/270.

10. Ikram, M., de Jong, F., Vingerling, J., Witteman, J., Hofman, A., Breteler, M., de Jong, P.: Are Retinal Arteriolar or Venular Diameters Associated with Markers for Cardiovascular Disorders? The Rotterdam Study. Investigative Ophthalmology \& Visual Science 45(7) (July 2004) 2129-2134

11. Wong, T.Y., Knudtson, M.D., Klein, R., Klein, B.E.K., Meuer, S.M., Hubbard, L.D.: Computer-assisted measurement of retinal vessel diameters in the Beaver Dam Eye Study: methodology, correlation between eyes, and effect of refractive errors. Ophthalmology 111(6) (June 2004) 1183-90

12. Leung, H., Hons, M., Jin, J., Mmed, W., Mapplstat, E.R., Hons, A.G.T., Frcs, T.Y.W., Mat, L.D.H., Mph, R.K., Franzco, P.M.: Clinical and Epidemiological Computer-assisted retinal vessel measurement in an older population : correlation between right and left eyes. Clinical and Exprimental Ophthalmology (2003) 326330 